\title{
Enseñanza de la fracción parte- todo, desde la resolución de problemas
}

\author{
Leidy Johana Molina Muñoz"
}

Artículo de reflexión

Fecha de Recepción: 23 julio 2018.

Fecha de Aprobación: 9 noviembre 2018.

\section{Resumen}

El estudio de las fracciones es uno de los ejes temáticos que más dificultad presenta para los estudiantes, debido a que al abordarlo no se tiene en cuenta el contexto real, lo cual puede causar que este concepto en su relación parte-todo sea poco comprendido. Desde esta perspectiva, este artículo presenta resultados de un proyecto de investigación cuyo objetivo es describir los alcances de una unidad didáctica que contribuya a la comprensión del concepto de fracción como parte de un todo a partir de las etapas de resolución de problemas propuestas por Polya (1982), para los estudiantes de $4^{\circ}$ de primaria de la Institución Educativa Antonio José
Sandoval Gómez, de Tunja. Para el desarrollo de esta investigación, se tuvo en cuenta el enfoque cualitativo y el tipo de Investigación Acción propuesta por Elliot (2000) y Kemmis y Mctaggart(1988) porque brinda la posibilidad de reflexionar sobre la práctica diaria, a partir de la observación directa (diarios de campo) con el propósito de implementar estrategias adecuadas que permitan el aprendizaje de los estudiantes.

Palabras clave: fracción parte-todo, resolución de problemas, unidad didáctica, investigación acción, comprensión, aprendizaje.
* Institución Educativa Antonio José Sandoval Gómez - Boyacá - Colombia leididy0910@hotmail.com

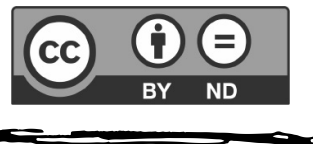




\section{Introducción}

A través de la revisión bibliográfica, se evidencia la dificultad que se presenta al enseñar el concepto de fracción, pues habitualmente los docentes siguen empleando metodologías conductistas que favorecen solamente la ejercitación algorítmica, omitiendo la construcción del concepto de una manera contextualizada y significativa. Así mismo, al predominar en las prácticas de aula un aprendizaje mecánico, el cual induce al estudiante a centrarse en la parte algorítmica, se limita la capacidad de desarrollar adecuadamente habilidades para la resolución de problemas y la comprensión de la fracción como parte de un todo. Se han desarrollado numerosas investigaciones respecto a este tema, tanto a nivel internacional como nacional, como por ejemplo Arteta J.; Escudero R.; Rojas C.; Martínez R.; Jiménez M.; Garrido L.; \& Badillo E. (2012), Perera \& Valdemoros (2007) quienes afirman que: "Uno de los factores que posiblemente inciden en este proceso es la didáctica tradicional empleada en la enseñanza, la cual sería uno de los factores determinantes del ulterior aprendizaje del niño” (p. 210)

Es tarea nuestra ayudarles, por un lado, a apreciar la presencia de los conceptos matemáticos en general, y de las fracciones en particular, en lo que ven y en lo que oyen, y por otro, a integrar los procedimientos de razonamiento, resolución de problemas en su actividad cotidiana
Por otra parte, los docentes, aún, no incorporan en su enseñanza, estrategias que posibiliten el fortalecimiento de sus prácticas educativas, para una comprensión de la temática de fracción, por parte de los estudiantes.

Ahora bien, Llinares \& Sánchez (1988) plantean:
Es tarea nuestra ayudarles, por un lado, a apreciar la presencia de los conceptos matemáticos en general, y de las fracciones en particular, en lo que ven $y$ en lo que oyen, $y$ por otro, a integrar los procedimientos de razonamiento, resolución de problemas en su actividad cotidiana (p.33).

Por consiguiente, se deduce, la necesidad de que los estudiantes comprendan el significado de la fracción y sus diferentes interpretaciones, para luego resolver cualquier situación referente al tema, sin dificultad; a su vez, el docente tiene la responsabilidad de conducir y guiarlo en la construcción y aplicación del concepto. Al respecto, la comunidad académica ha hecho valiosos aportes con relación a las diversas interpretaciones existentes frente al concepto de fracción: relación parte-todo, medida, cociente, razón y operador (Llinares \& Sánchez, 1988). Este trabajo estudia la relación partetodo, porque de aquí parten las demás interpretaciones: "La idea de fracción aparece a partir de situaciones en que está implícita la relación parte-todo" (Llinares \& Sánchez, 1988, p.13).

Según estas observaciones, se hace necesario diseñar e implementar una unidad didáctica que propicie la comprensión del concepto de fracción como parte de un todo, desde las etapas de resolución de problemas propuestas por Polya y el manejo de material didáctico para los estudiantes de $4^{\circ}$ de primaria de la Institución Educativa Antonio José Sandoval Gómez. Cabe destacar la importancia de usar material pues según lo propuesto por Gaviria, G. (2016) 
se hace necesario contar con un aula de clases rica en materiales didácticos como fichas, dominós, tangrams, ábacos, bloques multibases, geoplanos, bloques lógicos, figuras geométricas, calculadoras, computadores, etc. pues estos se constituyen en importantes herramientas para el desarrollo y enriquecimiento del proceso de enseñanza-aprendizaje de los estudiantes (p. 108 )

\section{Antecedentes}

A continuación, se presentan resultados, de la revisión de investigaciones relacionadas con la fracción y la resolución de problemas. Para cada uno de ellos se especifica datos relacionados con el tema en cuestión, objetivo, metodología, resultados y principales conclusiones.

\section{Sobre las fracciones}

\section{Nivel Internacional}

Fandiño, M (2009) en su tesis doctoral titulada "Las fracciones, aspectos conceptuales y didácticos" en el Núcleo de Investigación Didáctica de Bologna (Italia), expone una amplia panorámica de los aspectos históricos, epistemológicos, conceptuales y didácticos, explicando por qué se presentan las dificultades en el aprendizaje de los números fraccionarios, haciendo una lista de los errores, concepciones equivocadas, obstáculos y dificultades, proponiendo al final, algunas ideas para superar esos obstáculos.

La investigación recoge las experiencias de la autora, así como la de muchos otros investigadores. La autora señala que el estudio resultó más largo de lo que había previsto, debido a la intensa búsqueda bibliográfica, a la recopilación de experiencias de maestros de varios países de América Latina, también de Italia, España, Eslovaquia, Chipre, Grecia y Suiza. Dentro de las observaciones y sugerencias generales señala, en la acción de enseñanzaaprendizaje, es necesario hacer explícitos los problemas, en lugar de ocultarlos. El estudiante debe hacerse cargo de su propio aprendizaje, tener un contacto directo con el saber enseñado para transformarlo e integrarlo a sus saberes y lograr usarlo en diferentes escenarios de la vida.

\section{Nivel Nacional}

Meza \& Barrios, (2010), en el trabajo titulado "Propuesta didáctica para la enseñanza de las fracciones" plantean la utilización del juego en el plano pedagógico y didáctico, para desarrollar habilidades en los estudiantes que les permitan redescubrir las estructuras matemáticas. La pregunta generadora que orientaba esta investigación fue: ¿Cómo se puede lograr una mejor comprensión del concepto de fracción? Trataron de responder, a través de una estrategia didáctica de intervención en el aula, en la cual se toma el juego, con las regletas, para revisar el conocimiento del concepto de fracción en su forma verbal, simbólica y abstracta, confrontando a su vez el aprendizaje de la suma de fracciones mediante dos métodos específicos: el tradicional y un juego experimental, con la finalidad de obtener información sobre el grado de aceptación o preferencia de 
cada uno de los métodos señalados. Para la realización de este trabajo se seleccionaron cuarenta estudiantes del grado sexto de la Institución Educativa Alfonso Builes Correa del municipio de Planeta Rica, Córdoba, a quienes se les aplicó una prueba diagnóstica sobre el concepto de fracción, equivalencias y suma de fracciones, buscando indagar el grado de aprendizaje alcanzado por estos en el desarrollo de su programa de matemáticas, mediante el método de enseñanza tradicional. Luego se procedió a mostrarles otro camino que permitiera enfatizar los temas descritos anteriormente, a través del juego.

Después de haber desarrollado la propuesta, se indagó sobre la aceptación o preferencia de uno de los dos métodos señalados, concluyendo que el método del juego, es más fácil de manejar, más rápido y da un significado real. Además, se logra una mejor comprensión del concepto de fracción, un aprendizaje significativo debido al análisis y comprensión de las situaciones problema propuestas y no como producto de la repetición memorística de ejercicios. En este trabajo los autores señalan, que los estudiantes participaron activamente y con mucho entusiasmo, cuando sumaron fracciones a través del juego que les fue facilitado para tal fin, que cuando las realizaron por el método explicado tradicionalmente. De esta manera, se demuestra, que existen formas alternativas de enseñar matemáticas, las cuales favorecen el desarrollo del pensamiento matemático y contribuyen al mejoramiento de la calidad de la educación.
En el trabajo de grado de Prieto \& Vásquez, (2015) de la Universidad Distrital Francisco José de Caldas, titulado "Propuesta de una secuencia de actividades sobre interpretación de la fracción como parte- todo en contextos continuos y discretos, a partir de la propuesta de Sáenz (2010)" los autores, luego de realizar prácticas como docentes de matemáticas, percibieron que la enseñanza de las fracciones es uno de los temas difíciles de introducir en el aula de básica primaria. Además, plantean que el uso de las fracciones en la vida cotidiana es muy común, pues se observan situaciones en las que se debe repartir algo, ya sea en contexto continuo o discreto en tantas partes y este hecho se relaciona con el concepto de fracción. Por tanto, el docente debe hacer más énfasis en el concepto de fracción para desarrollar en los estudiantes la apropiación y reconocimiento de ésta.

De ahí surgió la pregunta de investigación ¿Qué estructura ha de tener una secuencia didáctica que contribuya a una interpretación de la fracción como parte todo en contextos continuos y discretos en estudiantes de grado cuarto, a partir de una adaptación realizada del trabajo de Sáenz, (2010)? Lo que se pretendía con la realización de este trabajo era: estructurar una secuencia de actividades basada en la propuesta de la profesora Sáenz, (2010) y los registros semióticos de Duval, (1999), para lograr la comprensión de la fracción parte todo, tanto en contextos continuos como discretos.

Para la realización de esta investigación, se tuvo en cuenta la metodología de la 
ingeniería didáctica propuesta por De Faria (2006), bajo el nivel de la microingeniería que tiene como fin el estudio de un determinado tema, que para este caso es la fracción y se tuvieron en cuenta, los resultados presentados por los estudiantes en el aula de clase al aplicar la diversas actividades para la comprensión del concepto de fracción.

Los instrumentos usados fueron: las entrevistas, los protocolos de clase en los que se registraba aspectos importantes como la pertinencia de las actividades y los obstáculos a los que se enfrentaban los niños a la hora de realizarlas. También se usó la observación participante, por cuanto cada situación planteada tuvo la intervención del docente practicante con el fin de orientar a los estudiantes, en el desarrollo de las tareas y lograr los objetivos propuestos.

Los autores concluyen que el aprendizaje de las fracciones es muy complicado, por tanto se recomienda al docente buscar las herramientas adecuadas para propiciarlo e incluir diferentes registros de representaciones. Se evidencia la necesidad de trabajar simultáneamente el contexto continuo y el discreto, pues no es suficiente abordar lo continuo y pensar, que con esto, el estudiante puede ser capaz de trasladarse al contexto discreto.

En el trabajo de grado de Echeverri \& Gutiérrez, (2014), para maestría, "Una propuesta didáctica para la enseñanza de los números fraccionarios" en enseñanza de las matemáticas de la Universidad de Antioquia, Medellín, se parte de la necesidad de desarrollar en los estudiantes habilidades y destrezas que le sirvan en la vida; por tanto, las autoras trabajaron una guía didáctica que permitiera dar pautas, para el trabajo de las fracciones a partir de la resolución de problemas cotidianos. Por eso se plantearon dos preguntas de investigación que orientaron la formulación de la propuesta didáctica: ¿Qué tipo de estrategia didáctica se puede implementar para la enseñanza de las fracciones para estudiantes de grado cuarto de educación básica primaria? ¿Son las situaciones de la vida cotidiana el mejor marco de referencia al momento de abordar las fracciones en el aula de clase con el objetivo de mejorar su asimilación?

El trabajo tuvo como fin, proporcionar estrategias y herramientas para que los educadores dinamicen sus prácticas de aula, partiendo de la contextualización y lograr que los estudiantes comprendieran y pudieran aplicar los conocimientos. Para alcanzar estos objetivos, iniciaron con un recorrido histórico del número fraccionario desde sus orígenes hasta la actualidad, enseguida explicaron las diferentes formas de representar un fraccionario, los campos de aplicación en diversos contextos, las dificultades que se observan tanto en los estudiantes como en los profesores a la hora de tratar el tema y finalmente mostraron la propuesta diseñada por las autoras, en la que presentan algunos recursos y materiales para la enseñanza del concepto de fracción. Además, incluyeron una guía apoyada en la resolución de problemas, la cual puede ser adaptada y trabajada en
En el trabajo de grado de Echeverri \& Gutiérrez, (2014), para maestría, "Una propuesta didáctica para la enseñanza de los números fraccionarios" en enseñanza de las matemáticas de la Universidad de Antioquia, Medellín 
cualquier institución. La guía, plantea situaciones problema, que el estudiante debe resolver con orientación del docente. Está diseñada para que sea resuelta bajo las cuatro etapas que propone Polya como son: comprensión del problema, concepción de un plan, ejecución del plan y visión retrospectiva (reconsideración de la solución). Las etapas tienen como guía, una serie de preguntas que permiten al estudiante encontrar posibles soluciones del problema.

Al finalizar el trabajo, concluyeron sobre la importancia de realizar un rastreo histórico de los conceptos, con los estudiantes, por cuanto lleva a entender la motivación por la que surgen los conceptos para relacionarlos con la vida diaria, por cuanto, para estudiar el concepto de fracción es conveniente partir del contexto cotidiano, esto posibilita la motivación e interés por el tema, presentándolos de forma sencilla, para que puedan ser manipulados por el estudiante. El docente tiene la responsabilidad de planear sus clases teniendo en cuenta el diario vivir, lo cual implica imaginación y creatividad, con el fin de adaptarlas para la explicación y fundamentación del trabajo en clase. Ahora bien, el llevar a los estudiantes por el paso a paso de (Polya, 1982) en la resolución de problemas, implica una estrategia metodológica que permite el análisis y reflexión; la puesta en común de los descubrimientos personales y los conocimientos aprendidos siendo una oportunidad valiosa para consolidar los conceptos trabajados.
Otro trabajo es el documento titulado "Los fraccionarios en primaria: retos, experiencias didácticas y alianzas para aprender matemáticas con sentido" de Arteta et al, (2012), en cuya obra se presenta la sistematización del desarrollo de la fase piloto del "Proyecto de mejoramiento de la enseñanza y el aprendizaje de las matemáticas" realizado por un grupo de quince escuelas de la ciudad de Barranquilla, cofinanciado por la Fundación ANDI y la Universidad del Norte en el año 2010. La pregunta que orientó el proceso fue ¿qué estrategias de intervención educativa dirigidas a los docentes de quinto de primaria pueden incidir en la transformación y el mejoramiento de la enseñanza y el aprendizaje de las matemáticas, de modo que se mejore el nivel de competencias de sus estudiantes? De acuerdo con este interrogante el programa se propuso como objetivo mejorar el conocimiento matemático y la formación didáctica de los maestros participantes para que adquirieran la capacidad de diseñar, aplicar y evaluar actividades significativas en el tema de fracciones, que posibiliten el desarrollo del pensamiento matemático en los estudiantes.

La metodología utilizada para el desarrollo de la propuesta fue la investigación acción con un enfoque del análisis cualitativo. Es así como se identifican cuatro fases: la primera es la diagnóstica, en la que se hace un reconocimiento de la institución educativa y la práctica pedagógica de los profesores participantes para indagar qué y cómo hacen las clases, además, se identifican las fortalezas y 
debilidades de los docentes respecto al pensamiento en matemáticas. Para el desarrollo de esta fase se realizan encuestas, entrevistas, filmaciones y análisis de clase. La segunda, es la fase de actualización desarrollada a través del seminario taller. En ésta, se hizo entrega de materiales a los participantes para la discusión, análisis, reflexión y problematización de las clases. En la tercera fase, denominada discusión de talleres se realizaron conferencias, intercambio de experiencias de aula y talleres para formar a los maestros. En la cuarta fase de innovación, los docentes participantes diseñaron propuestas de aprendizaje en las que se involucraba elementos conceptuales de números fraccionarios para estudiantes de quinto grado.

Respecto a las conclusiones se afirma: la motivación es un aspecto importante a tener en cuenta por parte del docente a la hora de orientar las clases, debe lograrse una actitud positiva hacia las matemáticas. Se hace necesario involucrar la resolución de problemas, ya que exige tiempo para pensar y explorar, puede incluir errores, los cuales se deben descubrir para volver a empezar, por tanto, los estudiantes adquieren el compromiso de no abandonar el problema. El docente debe estar en capacidad de trabajar problemas grandes o pequeños con una misma situación e incluir problemas relacionados con la vida real.

Otra de las conclusiones a las que se llegó, después de haber implementado secuencias didácticas que tenían como base el juego matemático para el desarrollo del concepto de fracción, es la motivación de los estudiantes durante el desarrollo de la actividad matemática y la construcción de significados colectivos.

Ahora bien, otro aspecto que juega un papel fundamental en el proceso de enseñanza y más aún en el tema de fracciones es la manipulación de material que apoya la comprensión de los conceptos. El maestro necesita estudiar a fondo, cada una de las tareas y el material a usar para que el alumno sea el protagonista; así, se mantiene el interés.

\section{Sobre Resolución de Problemas}

\section{A nivel Internacional}

Julca (2015) de la Universidad Privada Antenor Orrego de la ciudad de Trujillo (Perú) elaboró una tesis para optar por el título de Maestro en Educación, la cual se denominó "Uso del método Polya para mejorar la capacidad de resolución de problemas en matemática de los alumnos del primer grado de educación secundaria de la I.E. $N^{\circ} 81746$ Almirante Miguel Grau Seminario de Trujillo". Este trabajo de investigación buscaba demostrar que el uso del método de Polya mejora la capacidad para resolver problemas en matemáticas. Por lo anterior se planteó como pregunta de investigación: ¿En qué medida el uso del Método de Polya mejora la capacidad de resolución de problemas en matemáticas de los alumnos del primer grado de educación secundaria de IE $N^{\circ} 81746$ Almirante Miguel Grau Seminario de Trujillo? 
En el trabajo de pregrado realizado por Pérez (2015) de la Universidad Técnica de Ambato (Ecuador) titulado "El método Polya y el aprendizaje de la matemática en los estudiantes del cuarto año educación básica paralelo "D" de la unidad educativa Santa Rosa de la ciudad de Ambato provincia de Tungurahua" se hace énfasis en el proceso de enseñanza y aprendizaje que se desarrolla en matemáticas
Para el desarrollo de esta propuesta usó el diseño cuasi experimental en el que se tenían dos grupos: uno experimental $y$ otro de control. Inicialmente se aplicó una prueba a los dos grupos para establecer la capacidad de resolución de problemas, luego a uno de ellos se aplicó la propuesta pedagógica y el otro siguió con las tareas rutinarias. Al finalizar se volvió a aplicar un test a los dos grupos para establecer nuevamente la escala de calificación respecto a la resolución de problemas. Las sesiones para el grupo experimental se estructuraron con base en el Método Polya. En primer lugar, se hizo énfasis en la comprensión de lectura de los problemas, para pasar a la concepción de un plan, ejecución del plan en el que se tiene en cuenta la expresión de enunciados en lenguaje simbólico y por último la visión retrospectiva o comprobación de resultados.

Respecto a las conclusiones obtenidas se afirma que el grupo experimental mejoró significativamente en las habilidades para resolver problemas, en relación a las dimensiones de explorar, comprender, formular, planear, aplicar y reflexionar, lo que se evidencia a la hora de comprobar los promedios obtenidos por los dos grupos, pues al iniciar no había diferencia entre uno y otro. Además, se recomienda utilizar mayor tiempo para el uso del método Polya, específicamente en la dimensión ejecución de un plan, pues, muchas veces por cumplir con los contenidos programados, no se da el suficiente tiempo que necesitan los estudiantes. También es necesario verificar los conocimientos previos, para no tener problemas a la hora de aplicar el plan. Siempre se debe realizar autoevaluaciones entre profesor $y$ estudiantes sobre el desarrollo de la clase para establecer las fortalezas y falencias, así se podrá mejorar la aplicación del método.

En el trabajo de pregrado realizado por Pérez (2015) de la Universidad Técnica de Ambato (Ecuador) titulado "El método Polya y el aprendizaje de la matemática en los estudiantes del cuarto año educación básica paralelo "D" de la unidad educativa Santa Rosa de la ciudad de Ambato provincia de Tungurahua" se hace énfasis en el proceso de enseñanza y aprendizaje que se desarrolla en matemáticas, pues, es común encontrar problemas con un alto grado de dificultad para los estudiantes y en la mayoría de ocasiones no se presta la suficiente atención. Además, es usual ver que la enseñanza de las matemáticas se aborda con ejercicios mecánicos y de memorización donde se aplica una formula o procedimiento para obtener una respuesta. Ahora bien, este tipo de ejercicios no posibilitan el desarrollo del razonamiento, por tanto, se considera importante que el docente implemente la resolución de problemas como una medida que permita superar las dificultades señaladas.

Atendiendo a esta situación, surge la siguiente pregunta de investigación: ¿Influye el Método Polya en la enseñanza de las matemáticas en los estudiantes de la Unidad Educativa Santa Rosa de la Provincia de Tungurahua Cantón Ambato? Para 
responder a este interrogante, la autora tiene como propósito utilizar el Método Polya para construir una guía didáctica que permita mejorar el aprendizaje de las matemáticas en estudiantes de grado cuarto de básica Primaria. La metodología utilizada para el desarrollo del trabajo se basó en el enfoque cualitativo y cuantitativo; de acuerdo con esto, realizó una encuesta tanto a docentes como a estudiantes, que posteriormente se tabuló, analizó e interpretó. Es así como se evidencio la necesidad de implementar una guía metodológica que mejorara el proceso de enseñanza y aprendizaje del área de matemáticos, por cuanto se observó que ésta es impartida de una forma tradicionalista, no se usan métodos innovadores para motivar a los estudiantes, provocando el desinterés y descuido por parte de ellos. Luego de este análisis, se procedió a elaborar la guía didáctica para que los docentes pudieran apoyarse en ella, a la hora de orientar las clases de matemáticas en grado cuarto.

La investigación concluyó así: la aplicación del Método Polya mejora el aprendizaje de las matemáticas y conlleva a un trabajo dinámico y divertido; pero para aplicarlo es conveniente y necesario que los docentes lo conozcan a fondo, para que se sientan en confianza a la hora de usarlo y el provecho sea mayor. Así mismo, se sugiere que los maestros realicen un análisis sobre la enseñanza de las matemáticas, para identificar los intereses que tienen los estudiantes y lograr desarrollar todas sus habilidades en la materia. Finalmente se afirma que la propuesta presentada es de gran impacto porque mejora los aprendizajes, incentiva el interés y la comprensión por la asignatura y estimula la actividad mental en cada estudiante, cuando se trabaja de manera individual o grupal.

\section{Referentes teóricos}

A continuación, se presenta los dos ejes temáticos centrales que orienta el trabajo de investigación: la fracción parte-todo y la resolución de problemas. En relación al primer eje se establece recomendaciones por parte de investigadores para la enseñanza del concepto. Respecto al eje de resolución de problemas se describe las etapas propuestas por Polya, las cuales van a ser fundamentales para el desarrollo del trabajo.

\section{La Fracción}

Las investigaciones que se han desarrollado acerca del concepto de fracción recomiendan que para que el niño comprenda los temas se hace necesario plantear secuencias de enseñanza, con el fin de lograr en el estudiantado, una adecuada experiencia con las diversas interpretaciones de la fracción. Llinares (2003) plantea que en la enseñanza y aprendizaje de los números racionales, la dificultad para la adquisición de este concepto radica básicamente en que:

Están relacionados con diferentes tipos de situaciones (situaciones de medida, con el significado de parte de un todo, o como parte de un conjunto de objetos, de reparto utilizadas como cocien- 
te, como índice comparativo usadas como razón, y como un operador). $Y$, además, pueden representarse de varias maneras (3/4, fracciones; 75/100, fracciones decimales; 0.75 , expresiones decimales; 75\%, porcentajes) (p. 188).

En consecuencia, es cierto que para lograr aprendizaje significativo del concepto de fracción y sus diferentes interpretaciones, no solo en los estudiantes, sino también en los docentes de básica primaria, se requiere de un trabajo consciente y elaborado. Según Llinares \& Sánchez (1988) las interpretaciones que se deben trabajar con respecto al concepto de fracción son: La relación parte-todo y la medida, que se evidencia cuando el todo se divide en partes congruentes. Al trabajar esta interpretación se recomienda representar el todo en contextos continuos y discretos.

Otra de las interpretaciones corresponde, a la fracción como cociente, que es vista como la operación en la que se divide un número natural por otro. La fracción como razón es la interpretación que se refiere a una comparación entre dos cantidades de una magnitud. Finalmente, se presenta la fracción como operador, concebida como una sucesión de multiplicaciones y divisiones.

\section{Resolución de Problemas}

Ahora bien, es conveniente privilegiar el trabajo con situaciones problema, pues éstas proporcionan los contextos necesarios para conceptualizar la fracción; lo último, es el algoritmo, el cual es el resultado final de todo el proceso de evaluación de las estrategias personales. Además, es necesario que los estudiantes hagan asociaciones entre los modelos gráficos, la escritura, la lectura y la representación simbólica, evidenciando el concepto de fracción en su cotidianidad e interiorizando los procedimientos de razonamiento y de resolución de problemas en sus actividades diarias (Llinares y Sánchez, 1988).

Guzmán (como se citó en Ueno, 2014) comenta: lo que sobre todo se debe proporcionar a nuestros estudiantes a través de las matemáticas, es la posibilidad de hacerse un hábito de pensamiento adecuado para la resolución de problemas matemáticos y no matemáticos. ¿De qué les puede servir hacer un hueco en su mente en que se introduzcan unos cuantos teoremas y propiedades relativas a entes con poco significado, si luego van a dejarlos allí, herméticamente? A la resolución de problemas se le ha llamado, con razón, el corazón de las matemáticas, pues es ahí donde se puede adquirir el verdadero sabor que ha traído y trae a los matemáticos de todas las épocas (p. 161)

Por tanto, la resolución de problemas para los estudiantes es un proceso muy difícil, pues ellos están acostumbrados a realizar operaciones en forma intuitiva, con la esperanza de encontrar una respuesta acertada. En este caso, el maestro tiene la responsabilidad de orientar al estudiante, sabiendo que no lo debe abandonar, ni ayudarle demasiado; para que de esta manera pueda desarrollar una serie 
de habilidades que le permitan ser autónomo.

Para resolver problemas es necesario desarrollar como punto de partida, un proceso de exploración, buscando que el estudiante interiorice las estrategias personales que le permitirán dar solución a los diferentes problemas que se le planteen. Para abordar este tema, se tomará como referente, la teoría de resolución de problemas de Polya, (1982) pues en acuerdo con López (2008) "Pese a los años que han pasado desde la creación del método propuesto por Pólya, hoy día aún se considera como referente de alto interés acerca de la resolución de problemas" (p. 6). Ahora bien, es necesario llevar a cabo las cuatro etapas: comprender el problema, trazar un plan para resolverlo, poner en práctica el plan y comprobar resultados. A continuación, se explica este proceso:

En la comprensión del problema se determina el contexto, identificando los datos conocidos, desconocidos, los útiles y los distractores; abarcando así, la comprensión de la situación problema. En la etapa de trazar un plan para resolverlo se establece los caminos o estrategias que se tendrán en cuenta para llegar a la posible solución. En el siguiente paso, poner en práctica el plan, se aplica la estrategia seleccionada hasta lograr la solución del problema, este proceso es flexible pues conlleva a la reflexión y/o modificación de la estrategia. En la etapa final, comprobar resultados, se hace necesaria la comparación con el contexto del problema para poder determinar si los resultados coinciden; de no ser así, es necesario replantear el proceso.

Por otro lado, para adquirir la habilidad de resolver problemas, el estudiante requiere, desarrollar procesos metacognitivos que consciente $\mathrm{e}$ inconscientemente posibilitan un adecuado proceso de monitoreo del aprendizaje, pues según lo planteado por Mintzes, Wandersee \& Novak (1988),

La metacognición se refiere al conocimiento, concientización, control y naturaleza de los procesos de aprendizaje. En la metacognición el aprendizaje puede ser desarrollado mediante experiencias de aprendizaje adecuadas, cada persona tiene de alguna manera puntos de vista metacognitivos, algunas veces en forma inconsciente. De acuerdo con los métodos utilizados por los profesores durante la enseñanza, pueden alentarse o desalentarse las tendencias metacognitivas de los estudiantes (p. 4)

Además, Burón, J. (1996), plantea cuatro características fundamentales en la metacognición que están estrechamente relacionadas con la resolución de problemas y son:

Conocer los objetivos que se quieren alcanzar con el esfuerzo mental, elegir las estrategias para conseguir los objetivos planteados, observar el proceso de elaboración de conocimientos, para comprobar si las estrategias elegidas son las adecuadas, y evaluar los resultados para saber hasta qué punto se han logrado los objetivos.
La metacognición se refiere al conocimiento, concientización, control y naturaleza de los procesos de aprendizaje. En la metacognición el aprendizaje puede ser desarrollado mediante experiencias de aprendizaje adecuadas, cada persona tiene de alguna manera puntos de vista metacognitivos, algunas veces en forma inconsciente. 
La resolución de problemas es un proceso cognoscitivo complejo que involucra, el conocimiento almacenado en la memoria a corto y a largo plazo. Consiste en un conjunto de actividades mentales y conductuales, que implica factores de naturaleza cognoscitiva, afectiva y motivacional. (p. 15)

\section{Metodología}

Para el desarrollo de esta investigación, se tendrá en cuenta el tipo de Investigación Acción (IA), propuesto por Elliot (2000):

La investigación-acción se relaciona con los problemas prácticos cotidianos experimentados por los profesores, en vez de los "problemas teóricos" definidos por los investigadores puros, en el entorno de una disciplina del saber. Puede ser desarrollada por los mismos profesores o por alguien a quien ellos se lo encarguen (p. 24)

Por lo anterior, la IA, por sus características, facilita el estudio que se propone la investigación, al brindar la posibilidad de reflexionar sobre la práctica diaria a partir de la observación directa. En acuerdo a lo propuesto por Kemmis \& Mctaggart(1988) quienes también refieren la investigación acción, la investigación se realizó teniendo en cuenta las siguientes fases: planeación, acción - observación y reflexión.

En la fase de planeación, se diseñó una prueba diagnóstica con relación al concepto de fracción y la competencia de resolución de problemas, según lo planteado en los estándares; la cual buscaba establecer las fortalezas y debilidades de los estudiantes frente al concepto. Esta prueba estaba enfocada a confirmar los resultados presentados en las pruebas externas y fueron realizadas al inicio del estudio.

Además, en esta fase se definió un formato para los diarios de campo que fueron usados para registrar los hechos y experiencias en el aula de clase, durante la aplicación de la estrategia. También, se diseñó la unidad didáctica que consta de una secuencia de talleres basados en situaciones del contexto de los estudiantes y uso de materiales concretos para trabajar la fracción en su relación parte-todo en contextos continuos y discretos y la competencia de resolución de problemas. La unidad didáctica tiene elementos esenciales como: descripción, objetivos didácticos, contenidos de aprendizaje, secuencia de actividades, recursos, materiales, organización del espacio, tiempo y evaluación.

En la segunda fase (acción- observación) enfocada a la puesta en marcha de todo lo planeado en la fase anterior, se inició aplicando la prueba diagnóstica que permitió identificar los aspectos que se trabajarían en la unidad didáctica. Luego, se implementó la unidad en varias sesiones de clase, para consolidar en los estudiantes las cuatro etapas de resolución de problemas de Polya, desarrollando y afianzando la competencia de resolución de problemas con el concepto de fracción parte-todo y su uso. Para registrar los hechos y experiencias en el aula de clase, durante la aplicación de los 
talleres didácticos, se utilizó el diario de campo.

En la tercera fase (reflexión) se analizó, interpretó y concluyó aspectos relevantes para el proceso de investigación. Se identificaron las fortalezas, debilidades, aspectos por mejorar, estrategias empleadas por los estudiantes, competencias alcanzadas frente a la resolución de problemas y la comprensión del concepto de fracción en su relación parte- todo.

La técnica a utilizada para el análisis de los datos fue la triangulación porque permitió visualizar el problema desde diversos ángulos y diferentes métodos de recolección de datos como pruebas diagnóstico, diarios de campo $\mathrm{y}$ talleres que fueron codificados, analizados y comparados para validar la información. Además, de acuerdo a lo propuesto por Benadives M. y Gómez C. (2005) "La triangulación es una herramienta enriquecedora que le confiere a un estudio rigor, profundidad, complejidad y permite dar grados variables de consistencia a los hallazgos. A la vez permite reducir sesgos y aumentar la comprensión de un fenómeno.” (p. 123) Esto permitió una mayor validez de los resultados y una cercanía del investigador con el objeto de estudio.

\section{Resultados}

En la prueba diagnóstica se determinó que el $97 \%$ de los estudiantes no resuelve situaciones problema haciendo uso del concepto de fracción como parte de un todo. Además, se evidencio que ellos proceden a realizar una operación para dar respuesta a la situación, desconociendo el proceso de resolución de problemas. Por tanto, se trabajó el método de resolución de problemas, planteado por Polya, quien establece cuatro etapas. En la primera, comprender el problema, se generaron una serie de preguntas orientadoras como ¿cuál es la incógnita?, ¿cuáles son los datos? y ¿cuál es la condición? Esto, con el fin de que los estudiantes aborden los problemas con seguridad. Frente a la segunda etapa, concebir un plan, se plantearon preguntas como ¿necesita otros datos para determinar la incógnita? y ¿cómo solucionaría el problema? Para esta etapa se tuvo en cuenta los diversos esquemas de representación de los estudiantes, con el propósito de ayudarlos en la propuesta del plan de solución. En la tercera etapa, ejecución del plan, se hizo un seguimiento de cada uno de los pasos anteriores para comprobar si el problema estaba bien concebido, contestando las preguntas: ¿puede ver claramente que el paso es correcto? y ¿puede demostrar que es correcto? Finalmente, en la cuarta etapa, visión retrospectiva, el estudiante examinaba la solución obtenida, verificando el resultado y el razonamiento empleado con otros compañeros. En esta etapa los niños intercambiaban opiniones y sus posibles soluciones para luego establecer la más apropiada.

Respecto al concepto de fracción como parte de un todo es necesario aclarar que los problemas planteados se orientaron a esta temática. Además, los talleres realizados involucraron el juego como insumo de motivación y 
fácil comprensión de los conceptos para los estudiantes. La investigación mejoró significativamente la comprensión del concepto de fracción desde la resolución de problemas, superando las dificultades presentadas hasta el momento, convirtiendo al estudiante en un participante activo y también responsable de su proceso de aprendizaje.

\section{Conclusiones}

Es necesario orientar la unidad didáctica en torno a situaciones problema que tengan en cuenta los contextos cotidianos de los estudiantes para motivar y apropiar las etapas de resolución de problemas (comprender el problema, concebir un plan, ejecución del plan y visión retrospectiva) propuestas por George Polya (1982) en busca de las mejoras en el proceso de enseñanza y aprendizaje de los estudiantes de $4^{\circ}$ de primaria. Además, estas etapas evidencian un proceso riguroso aplicado antes de plantear la solución al problema, evitando que el estudiante de una respuesta rápida sin antes reflexionar para establecer la alternativa más adecuada.

Respecto a la fracción en su relación parte-todo se determina que los talleres propuestos enfocados a situaciones de la cotidianidad del estudiante son motivantes y propician un aprendizaje significativo, comprendiendo la utilidad del concepto en la vida diaria. Ahora bien, es necesario trabajar esta interpretación en contextos continuos y discretos como lo propone Llinares \& Sánchez, (1988) pues de aquí parten las demás interpretaciones del concepto de fracción.

En acuerdo con las investigaciones consultadas, se hace necesario iniciar por el cambio en el docente, quien debe ser capaz de autoevaluarse constantemente, romper con el esquema tradicional de la clase y generar ambientes de innovación en el quehacer, respondiendo a las necesidades de los estudiantes y la sociedad.

\section{Referencias}

Arteta J.; Escudero R.; Rojas C.; Martínez R.; Jiménez M.; Garrido L.; \& Badillo E. (2012), Los fraccionarios en primaria: retos, experiencias didácticas y alianzas para aprender matemáticas con sentido. Recuperado de: http://acofacien. org/images/files/BIBLIOTECA/Libros/LOS\%20FRACCIONARIOS\%20 EN\%20PRIMARIA.pdf

Benadives M. \& Gómez C. (2005). Métodos en investigación cualitativa: triangulación. Revista Colombiana de Psiquiatría, 34(1), 118-124.Recuperado de: http://www. redalyc.org/articulo.oa?id=80628403009 
Burón, J. (1996). Enseñar a aprender: Introducción a la metacognición. Bilbao, España. Mensajero.

De Faria E. (2006). Ingeniería didáctica. Cuadernos de Investigación y Formación en Educación Matemática.

Duval, R. (1999). Semiosis y Pensamiento Humano. Registros Semióticos y Aprendizajes Intelectuales. Cali, Colombia: Instituto de Educación y Pedagogía, Grupo de Educación Matemática.

Echeverri L \& Gutiérrez E (2014). Una propuesta didáctica para la enseñanza de los números fraccionarios. (Tesis de maestría) Recuperado de: http:// www2.udearroba.co/pluginfile.php/167290/mod_resource/content/2/ UNA \%20PROPUESTA \%20DIDACTICA \%20PARA \%20\%20LA $\% 20$ \%20ENSE\%C3\%91ANZA\%20DE\%20LOS\%20\%20NUMEROS\%20 FRACCIONARIOS.pdf.

Elliot, J. (2000). La investigación-acción en educación. Madrid, España: Morata.

Fandiño, M. (2009). Las Fracciones: aspectos conceptuales y didácticos. Bogotá, Colombia: Magisterio.

Julca, L. (2015). Uso del método Polya para mejorar la capacidad de resolución de problemas en matemática de los alumnos del primer grado de educación secundaria de la IE $N^{\circ} 81746$ almirante Miguel Grau seminario de Trujillo 2014. (Tesis de maestría) Recuperado de http://repositorio.upao.edu.pe/handle/upaorep/2296

Kemmis, S., \& Mc Taggart, R. (1992). Como Planificar la investigación-acción. Barcelona, España: Laertes S.A.

López, P. (2008). Estudio de la resolución de problemas matemáticos con alumnos recién llegados de Ecuador en Secundaria. (Tesis de doctorado). Recuperado de http:// www.tdx.cat/handle/10803/1328

Llinares, S., \& Sánchez, M. (1988). Fracciones: La relación parte-todo. Madrid, España: Síntesis.

Llinares, S., (2003). Didáctica de las Matemáticas para Primaria. Madrid, España: Pearson Educación S.A.

Mintzes, Wandersee, \& Novak, (1988). Aprendiendo a Aprender. Barcelona, España: Martínez Roca.

Ministerio de Educación, MEN, (2002). Decreto 1278. Bogotá. 
Meza, A., \& Barrios, A. (2010). Propuesta didáctica para la enseñanza de las fracciones. Ponencia presentada en $11^{\circ}$ Encuentro Colombiano Matemática Educativa. Bogotá, Colombia. Recuperado de http://asocolme.com/sitio/

Perera, P., \& Valdemoros, M. (2007). Propuesta didáctica para la enseñanza de las fracciones en cuarto grado de educación primaria. Investigación en Educación Matemática XI. Recuperado de: http://funes.uniandes.edu.co/1254/1/ Perera2008Propuesta_SEIEM_209.pdf

Pérez, H (2015). El método Polya y el aprendizaje de las matemáticas en los estudiantes del cuarto año educación básica paralelo " $D$ " de la Unidad Educativa Santa Rosa de la ciudad de Ambato provincia de Tungurabua (Tesis de pregrado).Recuperado de: http://repositorio.uta.edu.ec/jspui/handle/123456789/20244

Polya, G. (1982). Como plantear y resolver problemas. México: Editorial Trillas.

Prieto, D., \& Vásquez M. (2015). Propuesta de una secuencia de actividades sobre interpretación de la fracción como parte - todo en contextos continuos y discretos, a partir de la propuesta de Sáenz (Tesis de pregrado)Recuperado de: http://repository.udistrital.edu.co/bitstream/11349/2171/1/Prieto\%20 Hern $\%$ C3\%A1 ndez\%20Diana $\% 20$ Paola $\% 20 \% 20$ V\%C3\%A1squez $\% 20 \% 20$ Gonz\%C3\%A1lez\%20Maicol\%20Stiff\%202015.\%20PDF.pdf

Ueno, C (2014). Tiempo de (Video) Juegos. Números Revista de didáctica de las matemáticas, 86, 161-171. 